\title{
ALMOST PERIODIC SCHRÖDINGER OPERATORS II. THE INTEGRATED DENSITY OF STATES
}

\author{
JOSEPH AVRON AND BARRY SIMON
}

1. Introduction. In this paper, we will study Schrödinger operators, $-\Delta+V$, on $L^{2}\left(R^{\nu}\right)$ where $V$ is an almost periodic function on $R^{\nu}$. We will be especially interested in the case $\nu=1$ where we will also consider the finite difference analog on $l^{2}(\mathrm{Z})$

$$
(M u)_{n}=u_{n+1}+u_{n-1}+V(n) u_{n}
$$

where $V$ is an almost periodic function on $\mathbf{Z}$ (Jacobi matrix). Due to the recent discoveries of several workers including G. André, S. Aubry, M. Az’bel, J. Bellissard, V. Chulaevsky, E. Dinaburg, A. Gordon, D. Hofstader, R. Johnson, J. Moser, P. Sarnak, Ya. Sinai and D. Testard as well as the present authors (see the review [20]), it has become increasingly clear that these operators have subtle and fascinating spectral properties. In this paper, we study two technical objects - the integrated density of states (ids), $k(E)$, and the Lyaponov index, $\gamma(E)$, and their relation. The most interesting consequence of these developments is found in Section 7: Explicit, simple examples of Jacobi matrices, $M$, with purely singular continuous spectral measures.

Let us now describe $k$ in the Jacobi matrix case. Let $\mathscr{C}_{l}$ be the operator of multiplication by the characteristic function of $\{n \mid-l \leqslant n \leqslant l\}$. Then, we define

$$
k(E)=\lim _{l \rightarrow \infty}(2 l+1)^{-1} \operatorname{Tr}\left(\mathscr{C}_{l} P_{(-\infty, E)}(M)\right)
$$

where $\dot{P}_{\Omega}(\cdot)$ is the spectral projection for the operator $\cdot$ on the interval $\Omega$. We will prove the existence of the limit (2) in Section 2 in two steps: First, define the measure $d \mu_{l}$ by:

$$
\int f(E) d \mu_{l}=(2 l+1)^{-1} \operatorname{Tr}\left(\mathscr{C}_{l} f(H)\right)
$$

for continuous functions, $f$. We will show that $d \mu_{l}$ has a weak limit, $d k$, as $l \rightarrow \infty$. Secondly, we will show that $d k$ has no pure point piece from which (2) follows with

$$
k(E)=\int_{-\infty}^{E} d k
$$

Received June 25, 1982.

Research partially supported by USNSF Grant MCS 81-19979. 
by standard approximation arguments. We will show that both steps are true in the one dimensional Schrödinger case and the first step is true in higher dimension (it is quite likely the second is true then too, but we do not have a proof because we cannot bar infinite multiplicities of eigenvalues). One important property of $k$ that we will establish is that the spectrum of $M$ is the set of points of non-constancy of $k$, i.e.

$$
\operatorname{spec}(M)=\{E \mid k(E+\epsilon)-k(E-\epsilon)>0 \text { for all } \epsilon>0\}
$$

(3) is especially interesting since, as we will explain, we believe it can happen that $d k$ is disjoint from the spectral measure class. This is known to occur in other cases, e.g. for random potentials the ids is analytic while the spectrum is pure point.

In Section 3, we study continuity properties of $k$. The most interesting is the following: Let $k(E, \alpha)$ be the ids for $V(n)=\cos (2 \pi \alpha n)$. Then for $E$ fixed, $k$ is continuous at the irrational values of $\alpha$ and, in general, discontinuous at the rational values! This phenomenon holds for general a.p. functions. As the almost periods are varied, there are discontinuities at points where the dimension of the hull drops; i.e. where rational dependences of the almost periods occur.

The Lyaponov index, $\gamma(E)$, is defined as follows. Let $T_{l}(E)$ be the $2 \times 2$ matrix defined by

$$
T_{l}(E)\left(\begin{array}{l}
a \\
b
\end{array}\right)=\left(\begin{array}{l}
c \\
d
\end{array}\right)
$$

where $c=u(l+1), d=u(l)$ and $u$ is the solution of $M u=E u$ with $u(1)=a$, $u(0)=b$. Then

$$
\gamma(E)=\lim _{|l| \rightarrow \infty}|l|^{-1} \ln \left\|T_{l}(E)\right\|
$$

if the limit exists. In the context of random potentials, D. Thouless [23] found the remarkable relation

$$
\gamma(E)=\int \ln \left|E-E^{\prime}\right| d k\left(E^{\prime}\right)
$$

which we call the Thouless formula. Aubry-Andre [1], used the formula in the context of almost periodic Jacobi matrices. Thouless' proof of (5) is not rigorous. Indeed, the construction of Jacobi matrices with purely singular spectrum will rely on the fact that (5) may be false for $E$ in certain small sets, indeed for enough $E$ to support a spectral measure! What we will show using Thouless' argument and some functional analysis is that for almost all potentials $V_{w}$ in the hull of a fixed a.p. function, $V$, (5) holds for almost all $E$ with respect to Lebesgue measure. (5) is proven in Section 4. Its analog for the Schrödinger case appears in Section 5 .

In Section 6, 7 we restrict ourselves to what we will call the almost Mathieu 
equation, viz. (1), with

$$
V(n)=\lambda \cos (2 \pi \alpha n+\theta)
$$

where $\alpha, \theta, \lambda$ are parameters. In Section 6, we establish a rigorous symmetry found by Aubry [2], relating the operators for $(\lambda, \alpha)$ and for $(4 / \lambda, \alpha)$. In Section 7 , we prove that when $\alpha$ is irrational and $\lambda>2$, then for a.e. $\theta$, the operator has no absolutely continuous spectrum. This is also where we prove that for suitable $\alpha$, the spectrum is singular continuous. Section 8 discusses the implications of Section 7 for a class of Schrödinger operators which Bellissard et al. [4] showed were very close to the almost Mathieu equation.

We should say something about the connection of our work with that of Johnson-Moser [10]. While the methods are very different, there is considerable overlap with the results in Sections 2 and 5 and that part of Section 3 dealing with a fixed frequency module. They discuss an object which they call the rotation number (see also Moser [11]), which we shall see (in Section 2) is up to a constant identical to $k$. At the time we began our work on the ids we knew that they had proven that the rotation number existed but we didn't realize the equality of it and the ids. In any event, there is no doubt that their work on existence predates ours by several months. However, on the basis of discussions with Johnson, it is clear that the development of explicit properties was roughly parallel in time. They do not establish the Thouless formula, but they do discuss a closely related fact for $E$ with $\operatorname{Im} E>0$.

In comparing our methods with those in [10], we note the advantage of our method that at least for existence of $k(E)$. We can treat dimensions other than one dimension and moreover, we can treat the Jacobi matrix case. (It is possible that one can extend the Johnson-Moser method to the Jacobi case but the extension is not trivial). In favor of their rotation number approach, we mention the beautiful homotopy argument that "quantizes" $k(E)$ in gaps of the spectrum which we quote in Section 2 and which is proven by Johnson-Moser [10].

While such opinions are admittedly subjective, we feel our approach to existence of $k$ is simpler than that in [10]. One reason is that by thinking of rotation numbers, one cannot use the device of analyzing the weak limit $d k$ first and then prove continuity of the measure, $d k$.

We note that both we and [10] prove existence of $k$ for a fixed a.p. potential, $V$, and that this limit is the same for any other $W$ in the hull of $V$. If one is willing to settle for the slightly weaker result of existence and independence for a.e. $W$ in the hull (but perhaps not for the original, $V$ ), then the result is older and follows from a suitable application of the Birkhoff ergodic theorem. From the rotation number point of view, the result follows from arguments of Schwartzmann [17] and in terms of ids it is a result of Benderskii-Pastur [6] if we think of an a.p. function as an ergodic process. We also note that there is a third approach to study the properties of $k$ exploiting Von Neumann algebras. This approach is due to Shubin [18] and has been exploited by Bellissard-Testard [5]. 
We also note an interesting earlier paper of Scharf [16]. We prove here that $k$ exists by a simple argument once we know that the integral kernel $e^{-t H}(x, y)$ of $e^{-t H}$ obeys: $e^{-t H}(x, x)$ is an a.p. function for $t>0$. Our arguments would work just as well if one shows that $(H-z)^{-1}(x, x)$ is a.p. when $\operatorname{Im} z>0$. This is precisely what Scharf proves!

Throughout this paper we will use various standard properties of a.p. functions without comment. A sketch of the theory appears in the appendix to [3]. We will also use a few results from [3] about a.p. Jacobi matrices and a.p. Schrödinger operators. We do, however, want to note an alternative argument to the key fact that an a.p. function has an average. This fact, which will be the main input into the existence of $k$, is proven in [3] by exploiting the Birkhoff ergodic theorem and uniform continuity of the shift of the hull. A more elementary proof notes that for a finite linear combination of exponentials, the existence of $(1 / 2 T) \int_{-T}^{T} f(s)$ $d s$ is trivial, and any a.p. $f$ is a uniform limit of such combinations.

Next, we note a theorem of Gordon [8] which we will need in Section 7. His result is only given in the Schrödinger case but it has proof that is valid also in the Jacobi case. A description of his proof in English can be found in [20].

THEOREM 1.1J. Let $V$ be a function on the integers for which there exist functions $V_{m}$ periodic of period $T_{m} \rightarrow \infty$ so that

$$
\begin{gathered}
\sup _{m, n}\left|V_{m}(n)\right|<\infty \\
\sup _{-2 T_{m} \leqslant n \leqslant 2 T_{m}}\left|V_{m}(n)-V(n)\right| \leqslant m^{-T_{m}}
\end{gathered}
$$

Then any solution of $u(n+1)+u(n-1)+V(n) u(n)=E u(n)$ obeys

$$
\varlimsup_{|n| \rightarrow \infty}\left[u(n)^{2}+u(n-1)^{2}\right] /\left[u(1)^{2}+u(0)^{2}\right] \geqslant \frac{1}{4}
$$

and, in particular, no solution is in $l^{2}$.

THEOREM 1.1S. Let $V$ be a function on the real line for which there exist functions $V_{m}$ periodic of period $T_{m} \rightarrow \infty$ so that

$$
\begin{gathered}
\sup _{m, x}\left|V_{m}(x)\right|<\infty \\
\sup _{-2 T_{m} \leqslant x \leqslant 2 T_{m}}\left|V_{m}(x)-V(x)\right| \leqslant m^{-T_{m}}
\end{gathered}
$$

Then any solution of $-u^{\prime \prime}+V u=E u$ obeys

$$
\varlimsup_{|x| \rightarrow \infty}\left[u^{\prime}(x)^{2}+u(x)^{2}\right] /\left[u^{\prime}(0)^{2}+u(0)^{2}\right] \geqslant \frac{1}{4}
$$

and, in addition, no solution is in $L^{2}$. 
Finally, we note some terminology we will use for Diophantine properties of numbers. By a Liouville number, we mean an irrational number $\alpha$ for which there exist rational numbers $p_{k} / q_{k}$ with $q_{k} \rightarrow \infty$ and

$$
\left|\alpha-p_{k} / q_{k}\right| \leqslant k^{-q_{k}}
$$

An example is $\sum 2^{-a_{n}}$ where $a_{n+1}=n 2^{a_{n}}$ and $a_{1}=1$. The set of such $\alpha$ can be seen to be dense and uncountable but also of zero Lebesgue measure. Given real numbers $\left(\alpha_{1}, \ldots, \alpha_{l}\right)$ we say they have typical Diophantine properties, if there are constant $C, k$, so that for any integers $n_{1}, \ldots, n_{l}$, not all zero, we have

$$
\left|\sum n_{i} \alpha_{i}\right| \geqslant C\left(n_{1}^{2}+\cdots+n_{l}^{2}\right)^{-k} \text {. }
$$

The typical $n$-tuples have a complement with measure zero. But they are "non-generic" in the sense that they are of a second Baire category. The Liouville numbers have measure zero but a complement which has second category.

It is a pleasure to thank R. Johnson and P. Sarnak for useful discussions and to thank $\mathrm{Yu}$. Gordon for telling us of his work.

2. The integrated density of states. As a preliminary to showing that the integral kernel on diagonal, $e^{-t H}(x, x)$, is a.p. if $V$ is a.p., we want to prove some continuity of $e^{-t H}(x, y)$ in $V$. For this section, it would suffice to treat the case where $\left\|V_{n}-V\right\|_{\infty} \rightarrow 0$ but for the next section, the following stronger result is of interest. For the Jacobi matrix case we let $\left(M_{0} u\right)(n)=u(n+1)+u(n-1)$ and in the Schrödinger case $H_{0}=-\Delta$.

Proposition 2.1J. Let $V_{m}$ be a sequence of functions on $\mathbf{Z}$ obeying (i) $\sup _{n, m}\left|V_{m}(n)\right|<\infty$ (ii) $V_{m}(n) \rightarrow V(n)$ as $m \rightarrow \infty$ for some function $V$. Let $M=M_{0}+V ; M_{m}=M_{0}+V_{m}$. For an operator $A$ on $l_{2}$, let $A(i, j)(i, j \in Z)$ be its matrix elements. Then

$$
\lim _{m \rightarrow \infty}\left[\exp \left(-t M_{m}\right)\right](i, j)=[\exp (-t M)](i, j)
$$

for each $i, j$.

Proof. By hypothesis, $M_{m} \rightarrow M$ strongly so $\exp \left(-t M_{m}\right) \rightarrow \exp (-t M)$ strongly, so the matrix elements converge.

The analog in the Schrödinger case must be somewhat more subtle since even the existence of a continuous integral kernel is not completely trivial. We exploit Brownian motion results [19] but there is no question that other techniques could be used just as well. While our proof exploits boundedness of $V$, with more work one can handle suitable unbounded $V$ (see [21]). We will require the fact that there is for each $t>0$, a measure $d \mu_{0 ; x, y ; t}$ on continuous functions, $\omega$, from $[0, t]$ to $\mathrm{R}^{\nu}$ with $\omega(0)=\mathbf{x}, \omega(t)=\mathbf{y}$ so that $e^{-i\left(H_{0}+V\right)}$ has a continuous integral kernel 
for any bounded $V$ with

$$
\left(e^{-t\left(H_{0}+V\right)}\right)(x, y)=\int d \mu_{0 ; x, y ; t} \exp \left(-\int_{0}^{t} V(\omega(s)) d s\right)
$$

See [19] for a proof.

Proposition 2.1S. Let $V_{m}(x), V(x)$ be measurable functions on $\mathrm{R}^{\nu}$ so that (i) $\sup _{m, x}\left|V_{m}(x)\right|<\infty$ (ii) $V_{m}(x) \rightarrow V(x) m \rightarrow \infty$, for each $x$. Let $H=H_{0}+V$, $H_{m}=H_{0}+V_{m}$. Then for each fixed $x, y$ :

$$
\exp \left(-t H_{m}\right)(x, y) \rightarrow \exp (-t H)(x, y)
$$

Proof. Follows from (7) and the dominated convergence theorem.

In the above, we separated out the Jacobi and Schrödinger cases because the proofs were somewhat distinct. For the rest of this section and the next, the theorems and proofs are virtually identical so we only given them in the Schrödinger case (we note the few results that are special to this case). We also give the results only in 1-dimension, noting which results don't extend to higher dimension by saying "let $\nu=1$." We also note that while the Jacobi case was only given in 1-dimension, there is no bar to handling the discrete case in higher dimension to the same extent that the Schrödinger case in higher dimension can be handled.

THEOREM 2.2. Let $V$ be an almost periodic function on $R$ and let $\Omega$ be its hull. Given $w \in \Omega$, let $V_{w}$ be the associated potential. Let $H_{w}=H_{0}+V_{w}$. Then for any $w \in \Omega$ and $t>0$

$$
\lim _{l \rightarrow \infty}(2 l)^{-1} \operatorname{Tr}\left(\mathscr{C}_{(-l, l)}(x) e^{-t H_{w}}\right)=\mathscr{L}_{w}(t)
$$

exists and is independent of $w$. Moreover, for any $f$ in $L^{2}$ with $\|f\|_{2}=1$, we have that

$$
\mathscr{L}(t)=\int_{\Omega} d w \operatorname{Tr}\left(\tilde{f} e^{-t H_{w}} f\right)
$$

Proof. Fix $t>0$. Define

$$
g(w, x) \equiv\left(e^{-t H_{w}}\right)(x, x)
$$

Thus, if $w+x$ is defined by $V_{w+x}(y)=V_{w}(x+y)$, we have that

$$
g(w, x)=g(w+x, 0)
$$

If $w_{n} \rightarrow w$ in $\Omega$, then $V_{w_{n}}(x) \rightarrow V_{w}(x)$ uniformly in $x$, so by Prop. 2.1, $g\left(w_{n}, 0\right) \rightarrow g(w, 0)$. It follows that for $w$ fixed $g(w, x) \equiv h(x)$ is an almost periodic 
function with frequency module contained in $\hat{\Omega}$ the dual of $\Omega$. The existence of the limit of

$$
(2 l)^{-1} \operatorname{Tr}\left(\exp \left(-t H_{w}\right) \mathscr{C}_{(-l, l)}(x)\right)=(2 l)^{-1} \int_{-l}^{l} g(w, x) d x
$$

is just the existence of the average of an a.p. function. Thus, $\mathscr{L}_{w}(t)$ exists, is independent of $w$ and

$$
\mathscr{L}(t)=\int_{\Omega} d w g(w, 0)
$$

By the translation invariance of $d w$ and (8)

$$
\mathscr{L}(t)=\int_{\Omega} d w g(w, x)
$$

for any $x$, so

$$
\mathscr{L}(t)=\int_{\Omega} d w \int d x|f(x)|^{2} g(w, x)
$$

for any $f \in L^{2}$ with $\|f\|_{2}=1$. This is precisely the final assertion of the Theorem.

Let $C_{0}$ be the continuous functions on $R$ vanishing outside a compact set. A simple approximation argument (see [15]) exploiting the Stone-Weierstrass theorem, implies that

COROLlaRY 2.3. Under the above hypotheses on $V$,

$$
\mathscr{K}_{w}(F) \equiv \lim _{l \rightarrow \infty}(2 l)^{-1} \operatorname{Tr}\left(\mathscr{C}_{(-l, l)} F\left(H_{w}\right)\right)
$$

exists for any $F \in C_{0}$, is independent of $w$, and obeys

$$
\begin{aligned}
& \mathscr{K}_{w}(F) \geqslant 0 \quad \text { if } \quad F \geqslant 0 \\
& \mathscr{K}_{w}(F)=\int_{\Omega} d w \operatorname{Tr}\left(\bar{f} F\left(H_{w}\right) f\right)
\end{aligned}
$$

for any $f$ with $\|f\|_{2}=1$.

Now, fix $f$ with $f(x)>0$ for all $x$ and $\|f\|_{2}=1$. Define $d \mu_{w}$ to be the measure

$$
\int_{\Delta} d \mu_{w} \equiv \operatorname{Tr}\left(f P_{\Delta}\left(H_{w}\right) f\right)
$$

Let $d k$ ( $\equiv$ density of states measure) be the Borel measure with $\mathscr{K}_{w}(F)$ $=\int F(E) d k(E)$. Then

$$
d k=\int d w\left(d \mu_{w}\right)
$$


where (10) is short hand for

$$
\int F(E) d k(E)=\int d w \int F(E) d \mu_{w}(E)
$$

for $F$ continuous (this is just (9)). We have

Proposition 2.4. $d \mu_{w}$ is a spectral measure for $H_{w}$ in the sense that $P_{\Delta}\left(H_{w}\right)=0$ if and only if $\mu_{w}(\Delta)=0$.

Proof. A direct consequence of $f^{2}>0$.

THEOREM 2.5. $\operatorname{spec}\left(H_{w}\right)=\operatorname{support}(d k)$.

Remarks. 1. Recall [3] that $\operatorname{spec}\left(H_{w}\right)$ is $w$ independent.

2. We are not claiming that $d k$ is spectral measure for $H_{w}$. Indeed, this will only be true if the measure class of $d \mu_{w}$ is $w$ independent and this will be false if our belief is correct that $H_{w}$ can have dense point spectrum in suitable regions of energy. (For random potentials dense point spectrum is known to occur [7].)

Proof. If $F$ is a continuous function supported away from $\operatorname{spec}\left(H_{w}\right)$, then $F\left(H_{w}\right)=0$ for all $w$, so $\int F(E) d \mu_{w}=0$ for all $w$ and thus $\int F(E) d k$ is zero. Conversely if $F \geqslant 0$ and $F(E)>0$ for some $E \in \operatorname{spec}\left(H_{w}\right)$, then $F\left(H_{w}\right) \neq 0$, $F\left(H_{w}\right) \geqslant 0$ so since $f>0, \int F(E) d \mu_{w}(E)>0$ for all $w$. Thus $\int F(E) d k(E)>0$.

If we now define $k(E) \equiv \int_{(-\infty, E)} d k$, then Thm. 2.5 can be restated

Corollary 2.6. $\operatorname{Spec}\left(H_{w}\right)=\{E \mid$ for all $\epsilon>0, k(E+\epsilon)>k(E-\epsilon)\} \equiv$ the points of non-constancy of $k$.

Next we have an explicit formula for any possible discontinuity of $k$.

LEMMA 2.7. $\lim _{\epsilon \downarrow 0}\left[k\left(E_{0}+\epsilon\right)-k\left(E_{0}-\epsilon\right)\right]=\int_{\Omega} d w \operatorname{Tr}\left(f P_{\left\{E_{0}\right\}}\left(H_{w}\right) f\right)$ for any $f \geqslant 0$ with $\|f\|_{2}=1$.

Proof. Pick a monotone decreasing sequence of continuous functions $F_{m}(E)$ with $\lim _{m \rightarrow \infty} F_{m}(E)=0$ (resp 1) if $E \neq E_{0}\left(\right.$ resp $\left.E=E_{0}\right)$. Then

$$
\lim _{\epsilon \downarrow 0}\left[k\left(E_{0}+\epsilon\right)-k\left(E_{0}-\epsilon\right)\right]=\lim _{m \rightarrow \infty} \int F_{m}(E) d k
$$

and by the monotone convergence theorem

$$
\int d w \operatorname{Tr}\left(f F_{m}\left(H_{w}\right) f\right)=\int d w \operatorname{Tr}\left(f P_{\left\{E_{0}\right\}}\left(H_{w}\right) f\right) .
$$

All the results and proofs so far are valid for any dimensions. The following proof is special to one dimension.

LEMMA 2.8. Let $\nu=1$. Then the Haar measure of $\left\{w \mid E_{0}\right.$ is an eigenvalue of $\left.H_{w}\right\}$ is zero for each fixed $E_{0}$. 
Proof. (Following Pastur [13]) By translation invariance of $d w$,

$$
\int_{\Omega} d w \operatorname{Tr}\left(\mathscr{C}_{(l, l+1)} P_{\left\{E_{0}\right\}}\left(H_{w}\right)\right)=a
$$

independently of $l$. But $\sum_{l} \operatorname{Tr}\left(\mathscr{C}_{(l, l+1)} P_{\{E\}}\left(H_{w}\right)\right)=\operatorname{Tr}\left(P_{\{E\}}\left(H_{w}\right)\right)$. Since everything is positive, we can interchange sum and integral to obtain

$$
\int d w \operatorname{Tr}\left(P_{\left\{E_{0}\right\}}\left(H_{w}\right)\right)=a \cdot \infty
$$

with the convention $0 \cdot \infty=0$. Since $\operatorname{Tr}\left(P_{\left\{E_{0}\right\}}\left(H_{w}\right)\right) \leqslant 1$ for all $w, a$ must be zero. Thus $\operatorname{Tr}\left(P_{\left\{E_{0}\right\}}\left(H_{w}\right)\right)=0$ for a.e. $w$.

Remark. We believe this result is true in higher dimension. We regard it as the analog of Thomas' result [22] about absence of eigenvalues in the multidimensional periodic case.

The last two lemmas immediately imply the first assertion in the following:

THEOREM 2.9. Let $\nu=1$. Then $k$ is a continuous function of $E$ (called the ids). Moreover, for any $E$ and $w$ :

$$
\begin{aligned}
k(E) & =\lim _{l \rightarrow \infty}(2 l)^{-1} \operatorname{Tr}\left(\mathscr{C}_{(-l, l)} P_{(-\infty, E)}\left(H_{w}\right)\right) \\
& =\lim _{l \rightarrow \infty}(2 l)^{-1} \operatorname{Tr}\left(\mathscr{C}_{(-l, l)} P_{(-\infty, E]}\left(H_{w}\right)\right)
\end{aligned}
$$

Proof. As noted above, we must prove the final assertion. Let $d \mu_{l}$ be the measure given by $\int F(E) d \mu_{l}(E)=(2 l)^{-1} \operatorname{Tr}\left(\mathscr{C}_{(-l, l)} F\left(H_{w}\right)\right)$. Then $d k$ is the weak limit of $d \mu_{l}$. That

$$
k\left(E_{0}\right)=\lim _{l \rightarrow \infty} \int_{\left(-\infty, E_{0}\right)} d \mu_{l}(E)
$$

at points of continuity is a standard fact about weak limits.

Remark. Since weak limits of continuous measures can have pure points, one cannot obtain this result by only using $\left|(2 l)^{-1} \operatorname{Tr}\left(\mathscr{C}_{(-l, l)} P_{\{E\}}\left(H_{\nu}\right)\right)\right| \leqslant(2 l)^{-1} \rightarrow 0$.

For the one dimensional Schrödinger case, we link the ids to the rotation number of $[10,11]$ in a series of remarks, special to the one dimensional continuous case:

(1) Let $H_{w}^{(l)}$ denote the operator $-d^{2} / d x^{2}+V_{w}(x)$ on $(-l, l)$ with the boundary condition $u(-l)=u(l)=0$. Then, by a simple path integral argument, for $|x| \leqslant l$

$$
\left|\left[e^{-t H_{w}}-e^{-t H_{w}^{l}}\right](x, x)\right| \leqslant C(t) \exp \left[-D(t) d_{l}^{2}(x)\right]
$$

with $d_{l}(x)=\min (|x-l|,|x+l|)$, so by mimicking the above

$$
k(E)=\lim _{l \rightarrow \infty}(2 l)^{-1} \operatorname{Tr}\left(P_{(-\infty, E)}\left(H_{w}^{(l)}\right)\right)
$$


since the rhs is the sum of the dimensions of eigenfunctions, the term "density of states" is explained. We note that the analog holds easily for a wide class of other boundary conditions; indeed in one dimension, any b.c. which lead to $H_{w}^{(l)}$ which are uniformly bounded below (for difference of resolvents as b.c. change are at most rank 2).

(2) By standard o.d.e. techniques, if $u$ is the solution of $-u^{\prime \prime}+V_{w} u=E u$ with $u(-l)=0$, then

$$
\operatorname{Tr}\left(P_{(-\infty, E)}\left(H_{w}^{(l)}\right)\right)=\text { number of zeros of } u \text { in }(-l, l) .
$$

Then by Sturm comparison arguments, for any solution if $-u^{\prime \prime}+V_{w} u=E u$, we have

$$
\mid \operatorname{Tr}\left(P_{(-\infty, E)}\left(H_{w}^{(l)}\right)\right)-\text { number of zeros of } u \text { in }(-l, l) \mid \leqslant 1
$$

so that $k(E)$ is the density of zeros of $u$.

(3) If one looks at $\varphi(x)=u(x)+i u^{\prime}(x)$, and exploits Rolle's theorem, one sees that the density of zeros is $\pi^{-1}$ times the rate of the change of argument of $\varphi$, i.e.

$$
k(E)=\pi^{-1} \lim _{l \rightarrow \infty}(2 l)^{-1}[\arg \varphi(l)-\arg \varphi(-l)]
$$

This shows that $k(E)=\alpha(E) / \pi$ where $\alpha$ is the rotation number of JohnsonMoser. [Actually, J-M use the limit of $l^{-1}[\arg \varphi(l)-\varphi(0)]$ but this is easy to show, is equal to the limit of $(2 l)^{-1}[\arg \varphi(l)-\arg \varphi(-l)]$ by relating both to $k$.]

(4) Johnson-Moser use an elegant homotopy argument and this rotation number picture to prove that if $E \notin \operatorname{spec}\left(H_{w}\right)$, and if $\hat{\Omega}$ is normalized by requiring that $V_{w}$ is a limit of combinations of $\left\{e^{2 \pi i \alpha x}\right\}_{\alpha \in \hat{\Omega}}$, then

$$
k(E) \in \hat{\Omega}
$$

Bellissard-Testard [5] have an extension to the Jacobi matrix case by exploiting Connes' $C^{*}$-algebraic $K$-theory.

3. Continuity of the integrated density of states. In this section, we will study the dependence on $V$ of the density of states $k_{V}(E)$ for $-\left(d^{2} / d x^{2}\right)+V(x)$. Rather than prove directly continuity of $k_{V}(E)$ in $V$ for $E$ fixed, we will sometimes prove continuity of $\mathscr{L}_{V}(t)$ in $V$ for $t$ fixed whence as in the last section, continuity of $k$ in $V$ follows from the continuity in $E$. We first prove continuity if $V_{n} \rightarrow V$ in uniform norm. One could deduce this fairly easily from consideration of $\mathscr{L}$ since the integral kernel of $e^{-t H_{n}}$ converges uniformly to that of $e^{-t H}$. Instead, we proceed as follows:

LEMMA 3.1. (a) If $V \leqslant W$, then $k_{V}(E) \geqslant k_{W}(E)$.

(b) $k_{V+a}(E+a)=k_{V}(E)$

(c) $k_{V}(E) \leqslant k_{W}\left(E+\|V-W\|_{\infty}\right)$ 
Proof. (a) Obviously $H_{V}^{(l)} \leqslant H_{W}^{(l)}$ so that

$$
\operatorname{Tr}\left(P_{(-\infty, E)}\left(H_{V}^{(l)}\right)\right) \geqslant \operatorname{Tr}\left(P_{(-\infty, E)}\left(H_{W}^{(l)}\right)\right)
$$

and thus $k_{V}(E) \geqslant k_{W}(E)$.

(b) Follows from the obvious fact that

$$
P_{(-\infty, E+a)}\left(H_{V+a}^{(l)}\right)=P_{(-\infty, E)}\left(H_{V}^{(l)}\right) .
$$

(c) $W-\|V-W\|_{\infty} \leqslant V$, so using (a) and (b)

$$
k_{V}(E) \leqslant k_{W-\|V-W\|_{\infty}}(E)=k_{W}\left(E+\|V-W\|_{\infty}\right) .
$$

THEOREM 3.2. Let $\left\{V_{n}\right\}$ and $V$ be a.p. functions on $(-\infty, \infty)$. Suppose that $\left\|V_{n}-V\right\|_{\infty} \rightarrow 0$. Then for any $E$,

$$
k_{V_{n}}(E) \rightarrow k_{V}(E)
$$

Proof. By Lemma 3.1(c) and symmetry:

$$
k_{V}\left(E-\left\|V-V_{n}\right\|_{\infty}\right) \leqslant k_{V_{n}}(E) \leqslant k_{V}\left(E+\left\|V-V_{n}\right\|_{\infty}\right)
$$

so that continuity of $k_{V}(E)$ in $E$ implies the result.

Remark. In the $S$ case, one can read off the high energy behavior of $k_{V}(E)$ from Lemma 3.1(c) for it implies

$$
k_{V=0}\left(E-\|V\|_{\infty}\right) \leqslant k_{V}(E) \leqslant k_{V=0}\left(E+\|V\|_{\infty}\right)
$$

Since, in $\nu=1$

$$
k_{V=0}(E)=\pi^{-1} \sqrt{E}
$$

we see that

$$
\left|k_{V}(E)-\pi^{-1} \sqrt{E}\right|=O\left(E^{-1 / 2}\right)
$$

The other continuity result for $k$ that we wish to examine concerns the following. Fix $\nu$ and let $f$ be a fixed continuous function on the $\nu$-dimensional torus $T^{\nu}=\mathrm{R}^{\nu} / \mathrm{Z}^{\nu}$, i.e. $f$ is a continuous function on $R^{\nu}$ with $f\left(x_{i}+n_{i}\right)=f\left(x_{i}\right)$ if $n_{1}, \ldots, n_{\nu} \in Z^{\nu}$. (By combining our remarks below with the above considerations we could vary $f$ also as long as we required $\|\cdot\|_{\infty}$ convergence on $T^{\nu}$ ). For $\left(\alpha_{1}, \ldots, \alpha_{\nu} ; \theta_{1}, \ldots, \theta_{\nu}\right) \in R^{2 \nu}$, let $k(E ; \boldsymbol{\alpha}, \boldsymbol{\theta})$ be the density of states, for

$$
-\frac{d^{2}}{d x^{2}}+f\left(\alpha_{i} x+\theta_{i}\right) \equiv H(\boldsymbol{\alpha}, \boldsymbol{\theta})
$$

Remark. Since $k$ is constant over the hull of $V$ if the $\alpha_{i}$ are rationally independent, then $k(E ; \boldsymbol{\alpha}, \boldsymbol{\theta})$ is independent of $\theta$. We have the continuity results: 
TheOREM 3.3. Let $\bar{k}(E ; \alpha)=\int_{0}^{1} \cdots \int_{0}^{1} \prod d \theta_{i} k(E ; \boldsymbol{\alpha}, \boldsymbol{\theta})$. Then $\bar{k}$ is a continuous function of $\boldsymbol{\alpha}$.

Proof. Define $\mathscr{L}(t ; \boldsymbol{\alpha}, \boldsymbol{\theta})=\int e^{-t E} d k(E)$, so that we need only prove continuity of $\overline{\mathscr{L}}(t ; \boldsymbol{\alpha})=\int d \boldsymbol{\theta} \mathscr{L}(t ; \boldsymbol{\alpha}, \boldsymbol{\theta})$. But by Thm. 2.2:

$$
\bar{L}(t ; \boldsymbol{\alpha}, \boldsymbol{\theta})=\int d \boldsymbol{\theta} \operatorname{Tr}\left(\mathscr{C}_{1} e^{-t H(\boldsymbol{\alpha}, \boldsymbol{\theta})}\right)
$$

where $\mathscr{C}_{1}$ is the characteristic function of $(0,1)$ and by Prop. 2.1, (and the dominated convergence theorem in the $S$ case $), \operatorname{Tr}\left(\mathscr{C}_{1} e^{-t H(\alpha, \theta)}\right)$ is continuous in $\boldsymbol{\alpha}$ for $\boldsymbol{\theta}$ fixed. Thus, employing the dominated convergence theorem again, $\overline{\mathscr{L}}(t ; \alpha)$ is continuous in $\alpha$.

Remark. We emphasize that when one deals with the Jacobi case, the condition in the next theorem that $\alpha_{1}, \ldots, \alpha_{v}$ be rationally independent is replaced by " $\left(1, \alpha_{1}, \ldots, \alpha_{\nu}\right)$ must be rationally independent." This is precisely the condition needed for the orbit $\left\{\left(\alpha_{1} n, \ldots, \alpha_{n} n\right) \mid n=0, \pm 1, \ldots\right\}$ to be dense in $T^{\nu}$.

THEOREM 3.4. Let $\boldsymbol{\alpha}^{(\infty)}$ be a vector in $R^{\nu}$ of rationally independent frequencies. Let $\boldsymbol{\alpha}^{(k)} \rightarrow \boldsymbol{\alpha}^{(\infty)}$. Then for any $\theta^{(k)}, \theta^{(\infty)} k\left(E, \boldsymbol{\alpha}^{(k)}, \boldsymbol{\theta}^{k}\right) \rightarrow k\left(E, \boldsymbol{\alpha}^{(\infty)}, \boldsymbol{\theta}^{(\infty)}\right)$. (The last is independent of $\left.\theta^{(\infty)}\right)$.

Proof. By returning to the proof of Prop. 2.1, we see that

$$
\operatorname{Tr}\left(\mathscr{C}_{1} e^{-t H(\boldsymbol{\alpha}, \boldsymbol{\theta})}\right) \equiv g(\boldsymbol{\alpha}, \boldsymbol{\theta})
$$

is uniformly equicontinuous in $\boldsymbol{\theta}$ as we vary $\boldsymbol{\alpha}$. Now, by Thm. 2.2,

$$
\mathscr{L}\left(t, \boldsymbol{\alpha}^{(k)}, \boldsymbol{\theta}^{(k)}\right)=\int_{\Gamma^{k}} d \mu_{k}(\boldsymbol{\theta}) g\left(\boldsymbol{\alpha}^{(k)}, \boldsymbol{\theta}^{(k)}\right)
$$

where $\Gamma^{k}$ is the closure of $\left\{\boldsymbol{\theta}^{(k)}+R \boldsymbol{\alpha}^{(k)}\right\}$ and $d \mu_{k}$ is the obvious invariant measure. By the uniform equicontinuity, it suffices that $d \mu_{k}(\theta) \rightarrow d \mu$ weakly and this follows immediately from the rational independence of the $\alpha_{j}^{(\infty)}$.

When $\mathbf{a}_{j}$ are not independent, it is evident that $k(\boldsymbol{\alpha}, \boldsymbol{\theta})$ will have a non-trivial $\boldsymbol{\theta}$ dependence and thus since $k(\boldsymbol{\alpha}, \boldsymbol{\theta})$ is $\boldsymbol{\theta}$ independent when the $\alpha_{j}$ are independent, $k(\boldsymbol{\alpha}, \boldsymbol{\theta})$ can't be continuous in $\boldsymbol{\alpha}$ at points where the $\boldsymbol{\alpha}$ are rationally dependent, at least for most $\boldsymbol{\theta}$. Thus e.g. if $\nu=2$ and $\boldsymbol{\theta}=(0,0)$ is fixed, $k(1, \alpha)$ will typically be a function which is continuous at irrational $\alpha$ and discontinuous at rational $\alpha$.

As a detailed example of this phenomenon, let us consider the sequence of periodic potentials, $n=2,4, \ldots$.

$$
V_{n}(x)=\cos x+\cos \left[\left(1+n^{-1}\right) x\right]
$$

As $n \rightarrow \infty$, obviously $V_{n}$ converges pointwise to $2 \cos (x)$. On the other hand,

$$
V_{n}(x+\pi n)=\cos x-\cos \left[\left(1+n^{-1}\right) x\right]
$$


goes pointwise to the potential zero. In a gap for $2 \cos x, k(E)$ is constant while it is non-constant for $V=0$, so $k_{n}(E)$ cannot converge to both limiting $k(E)$ 's and presumably it converges to neither. By analyzing the spectrum of $H_{n} \equiv$ $-d^{2} / d x^{2}+V_{n}(x)$ further, one finds a rather subtle structure. First, we claim that the individual gaps in $\sigma\left(H_{n}\right)$ must be small. For if $\tilde{H}_{n}=-\left(d^{2} / d x\right)+$ $V_{n}(x+n \pi)=-\left(d^{2} / d x^{2}\right)-2 \sin (x / 2 n) \sin \left[x\left(1+2 n^{-1}\right)\right]$, then by choosing $u(x)$ $=e^{i k x} \varphi\left(x / n^{1 / 2}\right)$ with $\varphi \in C_{0}^{\infty}$, one sees that

$$
\|u\|_{2}^{2}=C_{1} n^{1 / 2} \text { and }\left\|\left(\tilde{H}_{n}-k^{2}\right) u\right\|_{2}^{2} \leqslant C_{2}(|k|+1)^{2} n^{-1 / 2}
$$

so $\left(k^{2}-D n^{-1 / 2}(|k|+1), k^{2}+D n^{-1 / 2}(|k|-1)\right) \cap \sigma\left(\tilde{H}_{n}\right) \neq \phi$. Thus if we look at any interval $[a, b] \subset(0, \infty)$, no gap can be larger than $O\left(n^{-1 / 2}\right)$ in size. On the other hand, each band for $H_{n}$ contributes $n^{-1}$ to the density of states so that as $n \rightarrow \infty$, the number of gaps in $[a, b]$ must approach $[n]\left\{\bar{k}_{\infty}(b)-\bar{k}_{\infty}(a)\right\}$, since the argument above shows that $k_{n}(E)$ approaches the average over $\theta$ of the $k(E)$ for $\cos x+\cos (x+\theta)$. Since these $k$ 's are all non-decreasing and $k(E)$ is strictly increasing on $(0, \infty)$ for $\theta=\pi, \bar{k}_{\infty}(b)-\bar{k}_{\infty}(a)>0$, i.e. we have many gaps, each of them small. Indeed, our guess is that in a gap for some $\cos x+\cos (x+\theta)$, one finds that for $n$ large, $H_{n}$ will have $n$ gaps of size $O\left(n^{-1}\right)$ and $n$ bands, each exponentially small.

There is one more continuity result on $k$ that we will require in Section 6 (we need it there in the Jacobi case; as usual, we state it in the Schrödinger case).

THEOREM 3.5. Let $\boldsymbol{\alpha}^{(\infty)}$ be a vector in $R^{\nu}$ of rationally independent frequencies. Let $\boldsymbol{\alpha}^{(k)} \rightarrow \boldsymbol{\alpha}^{(\infty)}$ be such that $V_{k}(x)=f\left(\alpha^{(k)} x\right)$ has a finite period $L_{k}$ (which automatically goes to $\infty$ as $k \rightarrow \infty)$. Let $H_{k}^{(P)}$ denote the Hamiltonian $-d^{2} / d x^{2}+V_{k}$ on $\left[0, L_{k}\right]$ with periodic boundary conditions. Then

$$
L_{k}^{-1} \operatorname{Tr}\left(P_{(-\infty, E)}\left(H_{k}^{(P)}\right)\right) \rightarrow k\left(E, \boldsymbol{\alpha}^{(\infty)}\right)
$$

Remark. At least for the proof we give it is critical that $L_{k}$ be periods, not just any numbers going to infinity.

Proof. For a periodic potential, $V$, of period $L$, we can compare $k(E)$ and $L^{-1} \operatorname{Tr}\left(P_{(-\infty, E)}\left(H^{(P)}\right)\right) \equiv \tilde{k}(E)$ quite easily. $\tilde{k}$ jumps by $L^{-1}$ at the bottom of the 1 st, 3 rd, . . . bands and at the tops of the 2 nd, 4 th, . . . bands. $k(E)$ increases in the bands by $L^{-1}$ in each band. Thus, for any $E,|k(E)-\tilde{k}(E)| \leqslant L^{-1}$. Thus

$$
\left|L_{k}^{-1} \operatorname{Tr}\left(P_{(-\infty, E)}\left(H_{k}^{(P)}\right)\right)-k\left(E, \alpha^{(k)}\right)\right| \leqslant L_{k}^{-1}
$$

so that this theorem follows from Thm. 3.4.

There is one final set of results about varying $\alpha$ that is of some interest.

THEOREM 3.6. If $\boldsymbol{\alpha}^{(\infty)}$ has rationally independent components and if $\boldsymbol{\alpha}^{k} \rightarrow \boldsymbol{\alpha}^{\infty}$, then $E_{\boldsymbol{e}} \in \operatorname{spec}\left(H\left(\boldsymbol{\alpha}^{(\infty)}, \boldsymbol{\theta}^{(\infty)}\right)\right)$ if and only if there exist $E_{k} \in \operatorname{spec}\left(H\left(\boldsymbol{\alpha}^{(k)}, \boldsymbol{\theta}^{(k)}\right)\right)$ with $E_{k} \rightarrow E_{\infty}$. 
Remark. The "only if" part is a general feature of strong resolvent convergence. The "if" part is special. The compactness of the hull essentially prevents eigenfunctions from "running away to $\infty$."

Proof. As remarked, we need only prove the "if" assertion. By a theorem of Johnson [9], $E_{k} \in \operatorname{spec}\left(H\left(\boldsymbol{\alpha}^{(k)}, \boldsymbol{\theta}^{(k)}\right)\right)$ implies that there exist bounded functions $u_{k}(x)$ and some other $\tilde{\theta}^{(k)}$ in the hull of $\boldsymbol{\theta}^{(k)}$, so that

$$
H\left(\alpha^{(k)}, \tilde{\theta}^{(k)}\right) u_{k}(x)=\left(E_{k} u_{k}\right)(x)
$$

by rescaling $u_{k}$ and translating $x$ (and $\theta^{(k)}$ ) we can suppose that $\left\|u_{k}\right\|=1$ but $u_{k}(0)>\frac{1}{2}$. By a simple estimate of $u_{k}^{\prime \prime}$, we see that $u_{k}(s)>\frac{1}{4}$ for $|s| \leqslant \delta$ for some small $\delta$ independent of $k$. By passing to a subsequence, we can be sure that $\tilde{\theta}^{(k)} \rightarrow \tilde{\theta}^{(\infty)}$ for some $\tilde{\theta}^{(\infty)}$ and that $u_{k}(x) \rightarrow u_{\infty}(x)$ pointwise (since the $u$ 's are uniformly equicontinuous). It follows that $u_{\infty}$ is a distributional eigenfunction of $H\left(\alpha^{(\infty)}, \tilde{\theta}^{(\infty)}\right)$ and it is non-zero since $u_{\infty}(x) \geqslant \frac{1}{4}$ on $(-\delta, \delta)$. Thus $E_{\infty}$ $\in \operatorname{spec}\left(H\left(\alpha^{(\infty)}, \tilde{\theta}^{(\infty)}\right)\right)=\operatorname{spec}\left(H\left(\alpha^{(\infty)}, \theta^{(\infty)}\right)\right)$.

Remark. Actually, one can prove Johnson's theorem by varying the above proof. If $E \in \operatorname{spec}\left(H\left(\alpha^{(\infty)}, \theta^{(\infty)}\right)\right)$ and we pick $\alpha^{(k)} \rightarrow \alpha^{(\infty)}$ with all $\alpha^{(k)}$ multiplies of a fixed $L^{-1}$ so $V\left(\alpha^{(k)}\right)$ is periodic, then by the "only if" part of the assertion, we can find $E_{k} \in \operatorname{spec}\left(H\left(\alpha^{(k)}, \theta^{(\infty)}\right)\right)$ with $E_{k} \rightarrow E$. In the periodic case, one knows that there are bounded eigenfunctions, so we can find $u_{k}$ with $H\left(\alpha^{(k)}, \theta^{(\infty)}\right) u_{k}=E_{k} u_{k}$ and then argue as above to prove Johnson's theorem without the need to appeal to the Sacker-Sell theory as he does.

By looking at the proof, one sees that even if the $\alpha$ 's are not independent, one can say something:

THEOREM 3.7. Let $S(\alpha)=\bigcup_{\theta} \operatorname{spec}(H(\alpha, \theta)$ ) (which by a simple argument is automatically closed). Then if $\alpha^{(k)} \rightarrow \alpha^{(\infty)}$, we have that $E_{\infty} \in S\left(\alpha^{(\infty)}\right)$ if and only if there exists $E_{k} \in S\left(\alpha^{(k)}\right)$ with $E_{k} \rightarrow E_{\infty}$.

Corollary 3.8. If $S(\alpha)$ is defined as above, then $\{(E, \alpha) \mid E \in S(\alpha)\}$ is a closed set.

4. The Thouless formula-Jacobi case. Let $T_{l, w}(E)$ be the transfer matrix defined in Section 1 for $M_{0}+V_{w}$.

Definition. We say that $(w, E)$ has Lyaponov behavior if and only if $\gamma_{w}(E)=\lim _{|l| \rightarrow \infty}|l|^{-1} \ln \left\|T_{l, w}(E)\right\|$ exists. $\gamma_{w}(E)$ is called the Lyaponov index.

The following is a standard consequence of the subadditive ergodic theorem (see [12]).

THEOREM 4.1. For each fixed $E, \gamma_{w}(E)$ exists for a.e. $w$ and is independent of w. $($ Call it $\gamma(E))$.

Remark. In Section 7, we will see explicit examples where $\{w \mid$ either $(w, E)$ hasn't Lyaponov behavior or $\left.\gamma_{w}(E) \neq \gamma(E)\right\}$ is non-empty. 
A direct argument shows that $\{(w, E) \mid(w, E)$ has Lyaponov behavior $\}$ is measurable, so by Fubini's theorem:

Corollary 4.2. A.e. pair $(w, E)$ has Lyaponov behavior.

Following Thouless, we proceed to try to compute $\gamma$. Notice that

$$
T_{l, w}(E)=\left(\begin{array}{cc}
E-V_{w}(l) & -1 \\
1 & 0
\end{array}\right) T_{l-1}, w(E)
$$

and thus by a direct induction argument for $l \geqslant 2$ :

$$
T_{l, w}(E)=\left(\begin{array}{cc}
P_{l, w}(E) & Q_{l-1, w}(E) \\
P_{l-1, w}(E) & Q_{l-2, w}(E)
\end{array}\right)
$$

where $P_{j, w}$ and $Q_{j, w}$ are monic polynomials of the degree $j$, i.e. $P_{j}(E)=E^{j}+\cdots$ Note $P_{l}(E)=0$ if and only if $H u=E u$ has a solution with $u(0)=0, u(l+1)=0$ and $Q_{l}(E)=0$ if and only if $H u=E u$ has a solution with $u(1)=0, u(l+2)=0$. Thus

$$
\begin{aligned}
& P_{l}(E)=\prod_{j=1}^{l}\left(E-E_{j}^{(l)}\right) \\
& Q_{l}(E)=\prod_{j=1}^{l}\left(E-\tilde{E}_{j}^{(l)}\right)
\end{aligned}
$$

where $E_{j}^{(l)}\left(\operatorname{resp} \tilde{E}_{j}^{(l)}\right)$ are the eigenvalues of $H$ with the b.c. $u(0)=u(l+1)=0$ (resp $u(1)=u(l+2)=0$ ). Letting

$$
d k^{(l)}=l^{-1} \sum_{j} \delta\left(E-E_{j}^{(l)}\right) d E
$$

and similarly for $\tilde{k}$ and letting

$$
\begin{gathered}
\gamma^{(l)}(E)=l^{-1} \ln \left|P_{l}(E)\right| \\
\tilde{\gamma}^{(l)}(E)=l^{-1} \ln \left|Q_{l}(E)\right|
\end{gathered}
$$

we see that

$$
\gamma^{(l)}(E)=\int \ln \left|E-E^{\prime}\right| d k_{l}^{(l)}\left(E^{\prime}\right)
$$

and similarly for $\tilde{\gamma}$. Since $d k^{(l)} \rightarrow d k$ weakly, if $\ln \left|E-E^{\prime}\right|$ were continuous, we could freely interchange $l \rightarrow \infty$ and the integral in (12) (which is what Thouless does). In fact, as we shall see in Section 7, this interchange is not allowed for all $E$ in general. However,

LEMMA 4.3. Let $\hat{\gamma}(E)=\int \ln \left|E-E^{\prime}\right| d k\left(E^{\prime}\right)$. Then for each fixed $w, \gamma^{(l)}(E)$ $\rightarrow \hat{\gamma}(E)$ in $L^{2}(d E)$ and the convergence is uniform in $w$. 
Proof. Since $k^{(l)}-k$ has compact support, we have that

$$
\gamma^{(l)}(E)-\hat{\gamma}(E)=\mathscr{P} \int\left[E-E^{\prime}\right]^{-1}\left[\left(k^{(l)}-k\right)(E)\right] d E
$$

Now $k^{l}-k$ goes to zero pointwise, so in $L^{2}$ by dominated convergence. (13) says that $\gamma^{(l)}-\gamma$ is the Hilbert transform of $k^{(l)}-k$ up to a factor of $\pi$. Thus, by the $L^{2}$ continuity of the Hilbert transform, $\gamma^{l} \rightarrow \gamma$ in $L^{2}$. The uniformity in $w$ follows from the uniformity of the convergence of $\mathscr{L}_{w}$ to $\mathscr{L}$.

Now pick a subsequence, $l_{k}$ so that for all $w$ :

$$
\left\|\gamma^{\left(l_{k}\right)}(E)-\gamma^{\left(l_{k+1}\right)}(E)\right\|_{2} \leqslant 2^{-k}, \quad\left\|\tilde{\gamma}^{\left(l_{k}\right)}(E)-\tilde{\gamma}^{\left(l_{k+1}\right)}(E)\right\| \leqslant 2^{-k}
$$

By the proof of the Reisz-Fisher theorem, off the measurable set of measure zero of pairs $(w, E)$ where either $\sum_{k}\left|\gamma^{\left(l_{k}\right)}(E)-\gamma^{\left(l_{k+1}\right)}(E)\right|^{2}=\infty$ or $\sum_{k} \mid \tilde{\gamma}^{\left(l_{k}\right)}(E)-$ $\left.\tilde{\gamma}^{\left(l_{k+1}\right)}(E)\right|^{2}=\infty$, we have that $\gamma^{\left(l_{k}\right)}(E) \rightarrow \hat{\gamma}(E), \tilde{\gamma}^{\left(l_{k}\right)}(E) \rightarrow \hat{\gamma}(E)$ and so

$$
\left|l_{k}\right|^{-1} \ln \left\|T_{l_{k}}(E)\right\| \rightarrow \hat{\gamma}(E)
$$

But, by Cor. 4.2, off another set of measure zero, the limit exists without passing to a subsequence. We conclude the following which is the major result of this section:

THEOREM 4.4. There is a set of measure zero, $Z$, of pairs $(w, E)$ so that for $(w, E) \notin Z$, we have that $(w, E)$ has Lyaponov behavior with

$$
\gamma_{w}(E)=\int \ln \left|E-E^{\prime}\right| d k\left(E^{\prime}\right)
$$

Remarks. 1. Since $k$ is only proven to be continuous, the integral in (14) is only known to converge a.e. in $E$. But the assertion is only claimed a.e. in $E$.

2. Without any significant change, the proof here extends to the case of general random Jacobi matrices with ergodic potentials.

5. The Thouless formula-Schrödinger case. In the Schrödinger case, we define the transfer matrix $T_{x, w}(E)$ as follows. Let $u, v$ solve the equation $-\varphi^{\prime \prime}+\left(V_{w}-E\right) \varphi=0$ with the b.c. $u(0)=0, u^{\prime}(0)=1 ; v(0)=1, v^{\prime}(0)=0$ and let

$$
T_{x, w}(E)=\left[\begin{array}{cc}
v(x, E) & u(x, E) \\
\frac{\partial v}{\partial x}(x, E) & \frac{\partial u(x, E)}{\partial x}
\end{array}\right]
$$

so $T_{x, w}(E)\left(\begin{array}{c}a \\ b\end{array}\right)=\left(\begin{array}{c}c \\ d\end{array}\right)$ means that the solution with $\varphi(0)=a, \varphi^{\prime}(0)=b$ has $\varphi(x)=c$, $\varphi^{\prime}(x)=d$. As in the Jacobi case, we say that $(w, E)$ has Lyaponov behavior if $\gamma_{w}(E)=\lim _{|l| \rightarrow \infty}|l|^{-1} \ln \left\|T_{l, w}(E)\right\|$ exists. Our goal here is to sketch the proof of the following: 
THEOREM 5.1. Let $\gamma^{(0)}(E)=[\max (0,-E)]^{1 / 2}$ and $k_{0}(E)=\pi^{-1}[\max (0, E)]^{1 / 2}$. For any a.p. function $V$ on $(-\infty, \infty)$, we have that for a.e. $E, \lim _{R \rightarrow \infty} \int_{-\infty}^{R} \ln E-$ $E^{\prime} \mid d\left(k-k_{0}\right)\left(E^{\prime}\right)$ exists and for a.e. pair $(w, E)$, the pair has Lyaponov behavior with $\gamma$ given by:

$$
\gamma(E)=\gamma_{0}(E)+\int_{-\infty}^{\infty} \ln \left|E-E^{\prime}\right| d\left(k-k_{0}\right)\left(E^{\prime}\right)
$$

Remarks. 1. Our proof works also in the random case. All that is needed is the ergodicity and boundedness of $V_{w}$.

2. (15) really makes two assertions. First, that $\gamma-i \pi k$ is the boundary value of an analytic function in the upper half plane, and secondly enough about the growth of the function at infinity to justify (15). The $\gamma_{0}, k_{0}$ is a "subtraction" procedure. Other subtraction procedures could be used. Johnson-Moser [10] prove that $\gamma-i \pi k$ is the boundary value of an analytic function.

We prove Thm. 5.1 in 8 steps emphasizing that which differs from the Jacobi case:

(1) Reduction to $x$ integral. Rescale so that 1 is rationally independent of the frequency module and thus $x \rightarrow x+1$ is ergodic on the hull. By noting that it is easy to obtain uniform (in $w$ ) bounds on

$$
\sup _{|x-y| \leqslant 1}\left\|T_{x, w}(E) T_{y, w}(E)^{-1}\right\|
$$

since $V_{w}$ is bounded, we can reduce the $|x| \rightarrow \infty$ limit to the limit along integral values of $x$.

(2) Analog of Thm. 4.1. Because of the ergodicity along integers, Thm. 4.1 extends

(3) Control of the $R \rightarrow \infty$ limit. We want to control the $R \rightarrow \infty$ using (11). Note that for $E<a<b$

$$
\begin{aligned}
\int_{a}^{b} \ln \left|E-E^{\prime}\right| d\left(k-k_{0}\right)\left(E^{\prime}\right)= & \int_{a}^{b} \frac{1}{E^{\prime}-E}\left[k\left(E^{\prime}\right)-k_{0}\left(E^{\prime}\right)\right] d E^{\prime} \\
& +{ }_{a}^{b}|\ln | E^{\prime}-E \mid\left[k\left(E^{\prime}\right)-k_{0}\left(E^{\prime}\right)\right]
\end{aligned}
$$

so by (11), $\lim _{b>a \rightarrow \infty} \int_{a}^{b} \ln \left|E-E^{\prime}\right| d\left(k-k_{0}\right)=0$ so that the $R$-limit exists so long as $\int_{-\infty}^{E+1} \ln \left|E-E^{\prime}\right| d\left(k-k_{0}\right)$ exists and this will be true for a.e. $E$ by standard Hilbert transforms. By exploiting the estimate $k_{0}\left(E-\|V\|_{\infty}\right) \leqslant k(E) \leqslant k(E+$ $\|V\|_{\infty}$ ) which led to (11), one also obtains the following that we will need below:

$$
\begin{gathered}
\lim _{M \rightarrow \infty}\left[\int_{k\left(E^{\prime}\right) \leqslant M} \ln \left|E^{\prime}-E\right| d k\left(E^{\prime}\right)-\int_{k_{0}\left(E^{\prime}\right) \leqslant M} \ln \left|E^{\prime}-E\right| d k_{0}\left(E^{\prime}\right)\right] \\
\quad=\int_{-\infty}^{\infty} \ln \left|E^{\prime}-E\right| d\left(k-k_{0}\right)\left(E^{\prime}\right)
\end{gathered}
$$


(4) Control of $l^{-1} \ln u_{0}(l, E)$. Let $u_{0}(x, E)=\sin (x \sqrt{E}) / \sqrt{E}$. We claim that for a.e. $E$,

$$
\lim _{l \rightarrow \infty}|l|^{-1} \ln \left|u_{0}(l, E)\right|=\gamma_{0}(E)
$$

the limit being through the integers. For $E<0$, this is easy. For $E>0$, we note that for a.e. $E$, we have that

$$
|l \sqrt{E}+\pi j| \geqslant c|l|^{-m}
$$

for suitable $c, m$. Then $u_{0}(l \sqrt{E}) \geqslant c^{\prime}|l|^{-m}$ so the limit in question is 0 .

(5) Finite e.v. estimates. Let $E_{k}(l)$ be the e.v. of $-d^{2} / d x^{2}+V(x)$ on $[0, l]$ with vanishing b.c. Let $E_{k}^{(0)}(l)=(\pi k / l)^{2}$ be the corresponding e.v. of $V=0$. By general principles

$$
\left|E_{k}-E_{k}^{(0)}\right| \leqslant\|V\|_{\infty}
$$

(6) Product formula in finite volume. We claim that for $l$ fixed

$$
\frac{u(l, E)}{u_{0}(l, E)}=\prod_{k=1}^{\infty}\left[\frac{E-E_{k}(l)}{E-E_{k}^{(0)}(l)}\right]
$$

(16) implies the absolute convergence of the product in (17) as well as that in $\prod_{k=1}^{\infty} E_{k}^{(0)}(l) / E_{k}^{(V)}(l)$. Standard integral equation techniques show that for $l$ fixed, (a) $\lim _{E \rightarrow-\infty} u / u_{0}=1$ (b) $\left|u_{0}(l, E)\right| \leqslant C_{1}^{(l)} \exp \left(C_{2}(l) \sqrt{|E|}\right)$ for complex $E$. Thus, by Hadamard product formulas:

$$
\begin{gathered}
u(l, E)=c \prod_{k=1}^{\infty}\left(1-E_{k}^{-1} E\right) \\
u_{0}(l, E)=d \prod_{k=1}^{\infty}\left[1-\left(E_{k}^{(0)}\right)^{-1} E\right]
\end{gathered}
$$

From this and (16) (to justify some interchanges of product), we obtain (17) up to an overall constant

$$
c d^{-1} \prod_{k=1}^{\infty} E_{k}^{(0)} / E_{k}
$$

in front. Since $u / u_{0} \rightarrow 1$ as $E \rightarrow-\infty$, we see that this constant must be 1 .

(7) Uniform finite l bound on $R \rightarrow \infty$ limit. We use (16) to note that

$$
\lim _{M \rightarrow \infty} \sup _{l \geqslant 1} l^{-1} \ln \left[\prod_{k \geqslant M l}\left|\left[E_{k}-E / E_{k}^{(0)}-E\right]\right|\right]=0
$$


For

$$
\begin{aligned}
\prod_{k \geqslant M l}\left|E_{k}-E / E_{k}^{(0)}-E\right| & =\prod_{k \geqslant M l}\left|\left[1+\frac{E_{k}-E_{k}^{0}}{E_{k}^{(0)}-E}\right]\right| \\
& \leqslant \exp \left(\sum_{k \geqslant M l}\left|E_{k}^{(0)}-E\right|^{-1}\left|E_{k}^{(0)}-E_{k}\right|\right) \\
& \left.\leqslant \exp \left(\|V\|_{\infty} \sum_{k \geqslant M l} l^{2} /\left(\pi^{2} k^{2}\right)\right) \quad \text { (if } M \text { is large }\right) \\
& \leqslant \exp \left(l\|V\|_{\infty} \int_{M-l^{-1}}^{\infty} d x / \pi^{2} x^{2}\right)
\end{aligned}
$$

(8) Completion of the proof. Given the last estimate in step (3) and (18) to control the large $E$ behavior, we can use the argument in Section 4 and (17) to obtain that for a.e. $E$

$$
\lim _{l_{k} \rightarrow \infty}|l|^{-1} \ln \left|u(l, E) / u_{0}(l, E)\right|=\int \ln \left|E-E^{\prime}\right| d\left(k-k_{0}\right)\left(E^{\prime}\right)
$$

for a suitable subsequence $l_{k}$. By using (4), we obtain control on the limit of $|l|^{-1} \ln |u|$ and by similar control of $v, \partial u / \partial x, \partial v / \partial x$ of $l^{-1} \ln \left\|T_{l}\right\|$. By step (1) control along a subsequence implies the required control for a.e. pair $(w, E)$.

6. Aubry duality. Thus far, all our results hold for general a.p. $V$ 's. Here we want to prove a very special fact of the almost Mathieu equation, i.e. the Jacobi matrix with $V$ given by (6). This result was discovered by Aubry [2] (see also Aubry-Andre [1]), whose proof is not rigorous because no careful study of the continuity properties of $k$ in $\alpha$ was given. Since we have proven Thm. 3.5, we will be able to directly follow the Aubry-Andre [1] argument in our rigorous proof.

THEOREM 6.1. Let $k(\alpha, E, \lambda)$ be the integrated density of states for the Jacobi matrix with $V(u)=\lambda \cos (2 \pi \alpha n)$. Then for any irrational $\alpha$,

$$
k(\alpha, \lambda, E)=k(\alpha, 4 / \lambda, 2 E / \lambda)
$$

We begin with a lemma which "explains" why (19) holds.

LeMMA 6.2. Let $\alpha=p / q$ with $p$ relatively prime to $q$. Let $H_{q}(\alpha, \lambda)$ be the Hamiltonian on $l_{2}(0, \ldots, q-1)$ given by

$$
\left(H_{q}(\alpha, \lambda) u\right)(j)=u(j-1)+u(j+1)+\lambda \cos (2 \pi \alpha j) u(j)
$$

where $u(q)$ is interpreted as $u(0)$ and $u(-1)$ as $u(q-1)$. Then $H_{q}(\alpha, \lambda)$ is unitarily equivalent to $\lambda H_{q}(\alpha, 4 / \lambda) / 2$. 
Proof. Let $W: l_{2}(0, \ldots, q-1) \rightarrow l_{2}(0, \ldots, q-1)$ by

$$
(W u)(j)=\sum_{n=0}^{q-1} u(k) \exp (2 \pi i \alpha n j)
$$

Since $W$ interchanges the finite difference and cosine terms we have

$$
W H_{q}(\alpha, \lambda) W^{-1}=\frac{\lambda}{2} H_{q}(\alpha, 4 / \lambda)
$$

Proof of Theorem 6.1. Given $\alpha$ irrational, pick $p_{k} / q_{k} \rightarrow \alpha$ with $p_{k}$ relatively prime to $q_{k}$. Let $k_{q}((p / q), \lambda, E)$ be $q^{-1}$ times the trace of the spectral projection for $H_{q}(\alpha, \lambda)$ associated to $(-\infty, E)$. By Lemma 6.2

$$
k_{q}\left(\frac{p}{q}, \lambda, E\right)=k_{q}\left(\frac{p}{q}, 4 / \lambda, 2 E / \lambda\right)
$$

By Thm. 3.5, since $\alpha$ is irrational,

$$
k_{q}\left(\frac{p}{q}, \lambda, E\right) \rightarrow k(\alpha, \lambda, E)
$$

Thus (19) holds.

Remark. While $H_{q}(\alpha, \lambda)$ and $\lambda H_{q}(\alpha, 4 / \lambda) / 2$ are unitarily equivalent, it is presumably not true that for $\alpha$ irrational, $H(\alpha, \lambda)$ and $\lambda H(\alpha, 4 / \lambda) / 2$ are equivalent. Indeed, if $\alpha$ has typical diophantine properties and $\lambda<2$, it is believed $H(\alpha, \lambda)$ has purely a.c. spectrum while $H(\alpha, 4 / \lambda)$ has purely point spectrum.

COROLlaRy 6.3. If $\alpha$ is irrational, then

$$
\operatorname{spec}(H(\alpha, \lambda))=\frac{\lambda}{2} \operatorname{spec}(H(\alpha, 4 / \lambda))
$$

Proof. Follows from Thm. 2.5 and Thm. 6.1.

Corollary 6.4. Fix $\lambda$, and fix $\alpha$ irrational. Then for almost all $E, \theta, \theta^{\prime}$, both $M_{0}+\lambda \cos (2 \pi \alpha n+\theta)-E$ and $M_{0}+4 \lambda^{-1} \cos \left(2 \pi \alpha n+\theta^{\prime}\right)-2 E / \lambda$ have Lyaponov behavior with indices related by

$$
\gamma(\lambda, \alpha, E)=\gamma(4 / \lambda, \alpha, 2 E / \lambda)+\ln (\lambda / 2)
$$

Proof. Follows from Thms. 4.3 and 6.1.

7. Absence of absolutely continuous spectrum. We are now prepared to prove what are our most interesting results. We first combine Cor. 6.4 with an argument of Pastur [13]. 
THEOREM 7.1. Fix $\lambda>2$, $\alpha$ irrational. Then for almost all $\theta, M_{0}+$ $\lambda \cos (2 \pi \alpha n+\theta)=M(\theta)$ has no absolutely continuous spectrum.

Proof. By the BGK eigenfunction expansion [21] for any $\theta S_{\theta} \equiv\{E \mid M(\theta) u$ $=E u$ has a polynomially bounded solution $\}$ supports the spectral measure class. By (20), $\gamma \geqslant \ln (\lambda / 2)>0$, so by Thm. $4.4 S_{\theta}^{(1)}=\{E \mid M(\theta)-E$ either fails to have Lyaponov behavior or has index 0$\}$ has measure zero for a.e. $\theta$. If $M(\theta)-E$ has Lyaponov behavior, then, by a theorem of Osceledec [12] every solution of $M(\theta) u=E u$ grows or decays exponentially at both $+\infty$ and $-\infty$. Thus, if $u$ is polynomially bounded, it decays exponentially and so is in $l_{2}$. Therefore

$$
S_{\theta} \subset S_{\theta}^{(1)} \cup S_{\theta}^{(2)}
$$

where $S_{\theta}^{(2)}=\{E \mid E$ is an eigenvalue of $M(\theta)\}$ is countable. Thus, for a.e. $\theta, S_{\theta}$ has measure zero and therefore, the spectral measure has no a.e. piece.

Combining this with Theorem $1.1 \mathrm{~J}$, we find:

Corollary 7.2. If $\alpha$ is a Liouville number and $\lambda>2$, then, for a.e. $\theta$, $M_{\theta}+\lambda \cos (2 \pi \alpha n+\theta)$ has only singular continuous spectral measure.

Remarks. 1. Since the set of Liouville numbers is a dense $G_{\delta}$, the result of Bellissard-Simon [24] applies and one also knows generically that $\operatorname{spec}(M(\theta))$ is a Cantor set. We believe it is probably a positive measure Cantor set. Recall that the spectrum is closed so $\bar{S}_{\theta}$ may have positive Lebesgue measure even when $S_{\theta}$ has none.

2. On account of Gordon's theorem, there are no generalized eigenfunctions going to zero at infinity. This provides a counterexample to the folk wisdom that a.c. spectrum corresponds to generalized eigenfunctions which do not go to zero at infinity while s.c. to eigenfunctions going to zero but in a non- $l_{2}$ sense. Perhaps, it is true that in this case the average of $u$ goes to zero.

3. In particular, if $\alpha$ is a Liouville number, the set $S_{\theta}^{(1)}$ of Thm. 7.1, the set on which the Thouless formula fails is non-empty, and we see that our care with sets or measure zero was necessary. It is the suppression of such sets that was the Aubry-Andre error. Also, by Johnson's theorem, if $S^{(1)}=\left\{(E, \theta) \mid E \in S_{\theta}^{(1)}\right\}$, then $\left\{\theta \mid(E, \theta) \in S^{(1)}\right\}$ is non-empty for any $E \in \operatorname{spec}(M(\theta))$.

8. A Kroning penny model. Bellissard et al. [4] have noted an intimate connection between solutions of

$$
\left[-\frac{d^{2}}{d x^{2}}+V(x)\right] \psi(x)=E \psi(x)
$$

and

$$
\left(\left[M_{0}+\lambda \cos (2 \pi \alpha n+\theta)\right] u\right)(n)=E u(n)
$$


if

$$
V(x)=\sum \mu \cos (2 \pi \alpha n+\theta) \delta(x-n)
$$

and

$$
E=k^{2} ; \quad \epsilon=2 \cos k ; \quad \lambda=\mu[\sin k / k]
$$

Indeed [20], if $\psi$ solves $(21)$, then $u(n) \equiv \psi(n)$ solves $(22)$ and $\psi$ is in $L^{2}$ if and only if $u$ is in $l_{2}$, etc. By analyzing the equation (22) and using the fact that the map $(E, \mu) \rightarrow(\epsilon, \lambda)$ is non-singular, one has:

THEOREM 8.1. Fix $\alpha$ irrational. The operator (21) with $V$ given by (23) has the property that for a.e. $\mu$ and $\theta$, there is no a.c. spectrum in the region $\left\{E=k^{2}|| \mu \sin k / k \mid>2\right\}$. If $\alpha$ is a Liouville number, then in that region, any spectrum must be singular continuous.

Unfortunately, we do not know there is any spectrum in the region in question, although we certainly believe that there is some spectrum there.

Added Note. After the typing of this article, we received a preprint of $\mathrm{M}$. Herman (Ecole Polytechnic Preprint), dated subsequent to the appearance of our announcement (Bull. A.M.S. 6(1982), 81-86). Herman also discusses rotation number and Lyaponov exponent and, in particular, proves the bound $\gamma(E) \geqslant \ln (\lambda / 2)$ of $(20)$. His proof works for a more general class of a.p. $V$ 's (trigonometric polynomials).

\section{REFERENCES}

1. G. ANDRE AND S. AUBRY, Analyticity breaking and Anderson localization in incommensurate lattices, Ann. Israel Phys. Soc. 3(1980), 133.

2. S. AUBRY, The new concept of transition by breaking of analyticity, Solid State Sci. 8(1978), 264.

3. J. Avron AND B. Simon, Almost periodic Schrödinger operators: I. Limit periodic potentials, Comm. Math. Phys. 82(1982), 101-120.

4. J. Bellissard, A. Formoso, R. Lima AND D. Testard, A quasi-periodic interaction with a metal-insulator transition, Phys. Rev. B 26(1982), 3024.

5. J. Bellissard AND D. TestaRd, in prep.

6. M. BeNDERSKII AND L. PASTUR, On the spectrum of the one-dimensional Schrödinger equation with a random potential, Mat. Sb. 82(1970), 245-256.

7. Ya. Goldstein, S. Molchanov And L. Pastur, A pure point spectrum of the stochastic one-dimensional Schrödinger operator, Func. Anal. Pril 11(1977), 1.

8. A. YA. GoRdon, On the point spectrum of the one-dimensional Schrödinger operator, Usp. Math. Nauk. 31(1976), 257.

9. R. Johnson, The recurrent Hill's equation, J. Diff. Eqns., 46(1982), 165-193.

10. R. Johnson AND J. Moser, The rotation number for almost periodic potentials, Comm. Math. Phys. 84(1982), 403-438.

11. J. MoSER, An example of a Schrödinger operator with almost periodic potential and nowhere dense spectrum, Comm. Math. Helv. 56(1981), 198-224.

12. V. I. OsELEDEC, $A$ multiplicative ergodic theorem. Lyaponov characteristic numbers for dynamical systems, Trudy. Mosk. Mat. Obsc. 19(1968), 679. 
13. L. PASTUR, Spectral properties of disordered systems in one-body approximation, Comm. Math. Phys. 75(1980), 179.

14. D. Pearson, Singular measures in scattering theory, Comm. Math. Phys. 60(1978), 13.

15. M. ReEd AND B. Simon, Methods of Modern Mathematical Physics, I. Functional Analysis, Academic Press, 1975.

16. G. Scharf, Almost periodic potentials, Helv. Phys. Acta. 38(1965), 573-605.

17. S. Schwartzman, Asymptotic cycles, Ann. Math. 66(1957), 270-284.

18. M. Shubin, Density of states for self-adjoint elliptic operators with almost periodic coefficients, Trudy Sem. Petrovskii 3(1978), 243.

19. B. Simon, Functional Integration and Quantum Physics, Academic Press, 1979.

20. B. Simon, Almost periodic Schrödinger operators: A Review, Adv. in Appl. Math. 3(1982), 463-490.

21. B. Simon, Schrödinger semigroups, Bull. Am. Math. Soc., to appear.

22. L. ThомAs, Time dependent approach to scattering from impurities in a crystal, Comm. Math. Phys. 33(1973), 335-343.

23. D. THOuLESS, $A$ relation between the density of states and range of localization for one-dimensional random system, J. Phys. C 5(1972), 77-81.

24. J. Bellissard AND B. Simon, Cantor spectrum for the almost Mathieu equation, J. of Func Anal. (1982), 408-419.

Department of Physics, California Institute of Technology, Pasadena, California 91125. Simon also at Department of Mathematics. 